\title{
Simultaneous Design and Control Framework for Multi-segment Multi-addition Plug-flow Crystallizer for Anti-solvent Crystallizations*
}

\author{
Qinglin Su, Chris D. Rielly, Zoltan K. Nagy, member IEEE
}

\begin{abstract}
Tubular reactors, which are often assumed to behave as plug-flow reactors, have many applications in chemical reaction engineering, because of their narrow residence time distribution and ease of scaling-up. In the pharmaceutical industries, the requirements of fast development and scalable design have also made the tubular crystallizer a promising platform for continuous manufacturing and crystallization processes which are widely recognized as an emerging technology for pharmaceutical manufacturing which aims to replace conventional capital- and labor-intensive batch operations. However, the interaction of effects, such as supersaturation, seed loading, nucleation and crystal growth, tube configuration and mean residence time have not yet been fully understood and optimized, from a process systems engineering (PSE) perspective, to achieve the most promising product qualities, such as the crystal size distribution. In this study, standardized modules representing plug-flow crystallizer (PFC) segments are assembled into a multi-segment multi-addition plug-flow crystallizer (MSMA-PFC) to facilitate the versatile design and control of anti-solvent crystallization processes, in which the total number, locations, and distribution of anti-solvent addition are to be optimized. An anti-solvent crystallization system of paracetamol-acetone-water was used as an example to compare the performances of different crystallizer configurations operated under optimal design. It was noticed that the proposed design outperforms the previous designs in literature which considered equally-spaced anti-solvent additions. Furthermore, the possibility of replacing existing batch crystallizers by MSMA-PFC is also discussed.
\end{abstract}

\section{INTRODUCTION}

Crystallization is a common unit operation for separation and purification of nearly $90 \%$ of organic molecules in the pharmaceutical and fine chemical sectors [1, 2]. Traditionally, batch operation has been adopted for crystallization and the downstream secondary manufacturing processes to adjust to the stringent regulations in product quality and to the flexible demands of the market. However, rising market competitiveness and the need to reduce manufacturing costs, now drive the future of pharmaceutical and fine chemical industries towards continuous processes,

*Research supported by UK EPSRC ICT-CMAC funding

Q. $\mathrm{Su}$ is with the Chemical Engineering Department, Loughborough University, LE11 3TU UK (e-mail: Q.Su@lboro.ac.uk).

C. D. Rielly is with the Chemical Engineering Department, Loughborough University, LE11 3TU UK (phone: +44 (0)1509 222504; fax: +44 (0)1509 223 923; e-mail: C.D.Rielly@lboro.ac.uk).

Z. K. Nagy is with the School of Chemical Engineering, Purdue University, West Lafayette, IN 47907-2100 USA (phone: +1 (765) 494-0734; fax: +1 (765) 494-0805; e-mail: Z.K Nagy@purdue.edu). which have potential for improvements in quality control, equipment footprint, and energy and labor costs, etc., [3].

In the last decade, the development of continuous manufacturing and crystallization techniques has focused on changing from batch to continuous operation using existing unit operations $[4,5]$, in addition to studying innovative equipment design. For example, existing batch crystallizers based on stirred-tank designs can be converted to continuous mode as multi-stage mixed-suspension and mixed-product removal (MSMPR) operations [6]. In contrast, tubular designs of continuous crystallizers have become state-of-the-art techniques in recent years. Compared to conventional stirred-tanks, the potential benefits of tubular designs result from the narrow residence time distribution, and the ease of scaling-up for continuous crystallization processes.

For example, recent research efforts have been devoted to the experimental investigation of tubular crystallizers. Lawton et al. [7] reported the application of a continuous oscillatory baffled crystallizer (COBC) which offers significant advantages in operating cost and processing time. Eder et al. [8, 9] investigated the impacts of flow rate and seed loading in continuously-seeded tubular crystallizers for the conceptual production of active pharmaceutical ingredients. Alvarez et al. [1] showed that a distributed addition of antisolvent along the PFC has an effect on the crystal size distribution (CSD).

In addition, process systems engineering (PSE) approaches using mathematical modelling, intensification, and optimization are also applicable to the design of tubular crystallizers [10]. Majumder and Nagy [2] optimized the temperature profile along a multi-segment PFC, which included cooling and heating segments, with an aim to remove crystal fines by controlled dissolution. Vetter et al. [11] recently investigated the attainable regions of particle sizes for a single-stage ideal plug-flow crystallizer, which assumed continuous addition of anti-solvent along the tube; they compared the achievable product qualities to those manufactured in MSMPR and batch crystallizers. Moreover, Ridder et al. [12] introduced the general concept of multi-segment multi-addition PFC (MSMA-PFC) and proposed a simultaneous design and control (SDC) approach to optimize the number of segments and anti-solvent distributions along a MSMA-PFC for an anti-solvent crystallization processes. However, considering the various complexities in crystallization, such as the well-known effect of anti-solvent addition which may increase or decrease the supersaturation depending on the slope of the solubility curve, PSE still has a large part to play. For instance, to date, 
flowsheet simulations of plug-flow crystallizer are still not available in most commercial software packages, e.g., the gCRYSTAL 4.0 software offered by Process System Enterprises Ltd. Hence, it would be of interest to study the mathematical modelling, design, and optimization of tubular crystallizers for anti-solvent crystallization in order to provide a better understanding and flexible design, and to improve their performance accordingly.

The multi-segment multi-addition design of a plug-flow crystallizer is a general requirement for an anti-solvent crystallization to enable the accurate control of supersaturation and anti-solvent mass fraction profiles along the length of the tube. The modular design of PFC segments [1], where flexibility is a key aspect, is intended for campaign production of different active pharmaceutical ingredients (APIs) using the same line. This is an important consideration for the design of a continuous manufacturing process [13]. For example, varying lengths of tube would be possible by assembling a different number of PFC segments. Furthermore, distributions of anti-solvent among the segments can be optimized to accommodate different supersaturation profiles along the tube [12].

This paper is organized as follows. A steady-state mathematical model of a general plug-flow crystallizer is first presented, followed by its extension to a multi-segment multi-addition design for an anti-solvent crystallization processes. Unlike the equally-spaced anti-solvent addition proposed in [12], a mixed integer non-linear programming (MINLP) optimization is taken into account to optimize both the locations and distributions of anti-solvent additions along the tubular crystallizer. The results and discussion section considers the application of the proposed design and optimization framework to an anti-solvent crystallization of paracetamol-acetone-water system and compares its performance to that obtained by its counterpart. Finally, concluding remarks are made.

\section{Mathematical Modelling OF Plug-FlOW CRYSTALLIZER}

In the pharmaceutical industries, a typical bulk ingredient is usually produced at a rate of $>1$ ton per day [14] and thus the diameter of a tubular crystallizer could be around just several centimeters, which is small when compared to typical tube lengths (tens of metres). Therefore there is often near perfect mixing in the radial direction and limited dispersion in the axial direction. Moreover, a relatively high slurry mean velocity along the tube is required to avoid the sedimentation of crystals. (In the case of the oscillatory baffled crystallizer, high-frequency oscillations are introduced to suspend the crystals.) Therefore, it is often reasonable to assume that the tubular crystallizer is a plug-flow crystallizer and therefore requires only one spatial dimension, i.e. the tube axial length, in a mathematical model.

A steady-state model of a plug-flow crystallizer is obtained straightforwardly by considering a population balance equation for crystals and a mass balance equation for solute concentration, as shown below.

$$
\frac{\partial n}{\partial z}+\frac{1}{v_{z}} \frac{\partial(G n)}{\partial L}=0
$$

$$
\frac{d C}{d z}+\frac{3 \rho_{s} K_{v}}{v_{z} M_{w}} \int G L^{2} n d L=0
$$

where $n$ is the number density of crystals in the slurry, $\# \mathrm{~m}^{-3} ; z$ is the tube axial length, $\mathrm{m} ; v_{\mathrm{z}}$ is the slurry mean velocity along the tube axial, $\mathrm{m} \mathrm{s}^{-1} ; G$ is the crystal growth rate, $\mathrm{m} \mathrm{s}^{-1} ; L$ is the characteristic length of crystal, $\mathrm{m} ; C$ is the solute concentration, $\mathrm{kmol} \mathrm{m}^{-3} ; \rho_{s}$ is the crystal density, $\mathrm{kg} \mathrm{m}^{-3} ; K_{\mathrm{v}}$ is the volumetric shape factor of crystal; $M_{w}$ is the molecular weight of crystal, $\mathrm{kg} \mathrm{kmol}^{-1}$.

The slurry mean velocity $v_{z}$ along the crystallizer is not necessarily constant, e.g. if the volume changes due to crystallization (formation of solids) along the crystallizer cannot be neglected; otherwise $v_{z}$ will depend on the yield of crystals at each point along the crystallizer and should be rigorously calculated. In this study, $v_{z}$ is assumed to be a constant mean velocity along the tube [1] and there is no breakage or agglomeration in the tube.

The corresponding boundary conditions for $n(L, z)$ and $C(z)$ at $L=0$ or $z=0$ are listed as follows.

$$
\begin{gathered}
n(0, z)=B / G \\
n(L, 0)=n_{\text {seed }}(L) \\
C(0)=C_{\text {feed }}
\end{gathered}
$$

where $B$ is the nucleation rate, $\# \mathrm{~m}^{-3} \mathrm{~s}^{-1} ; n_{\text {seed }}$ is the seed crystal size distribution (CSD) at the crystallizer inlet, $\# \mathrm{~m}^{-3} ; C_{\text {feed }}$ is the feeding solute concentrations at the crystallizer inlet, kmol $\mathrm{m}^{-3}$

The above partial differential equation (PDE), eq.(1), can be solved in MATLAB with a high-resolution finite-volume method (FVM) [15] by discretizing the crystal characteristic length $L$ and then integrating the resulted ordinary differential equations (ODEs), together with eq.(2), along the tube length $z$ (method of lines). The FVM scheme is of second-order accuracy, combining a robust upwind discretization method with the novel $k=1 / 3$ flux limiter, capturing the sharp front of the nucleation boundary in eq.(3) without numerical oscillations, and providing a smooth solution. The integration of ODEs was executed with the built-in ode 45 or ode 23 functions for non-stiff problem in MATLAB.

Depending on the fineness of discretization in the FVM, the PDEs often result in tens or hundreds of ODEs which can be computationally expensive to solve. Alternatively, when the crystal growth rate $G$ is size independent, the classical method of moments (MOM) can also be applied to integrate the eq.(1) along the crystal length $L$ by conversion into a set of moment ODEs, which further reduces the computational burden for steady-state modelling. This is critically important when a large optimization problem is considered. In the MOM, the integration and moment transformation of the PDE into the first six moments are given below.

$$
\begin{gathered}
\mu_{k}=\int_{0}^{\infty} L^{k} n(L, z) d L, \quad k=0 \cdots 5 \\
\frac{d \mu_{0}}{d z}=\frac{B}{v_{z}}
\end{gathered}
$$




$$
\frac{d \mu_{k}}{d z}=k\left(\frac{G}{v_{z}}\right) \mu_{k-1}+\left(\frac{B}{v_{z}}\right) L_{0}^{k}
$$

where $\mu_{k}$ is the $k^{\text {th }}$ moment of the crystal size distribution $n$, $\mathrm{m}^{\mathrm{k}} \mathrm{m}^{-3} ; L_{0}$ is the nuclei size, $\mathrm{m}$. Although the full CSD development along the tube $z$ is lost by the integration of eq.(6), the physical meanings of the first few moments provide useful information: e.g. $\mu_{0}$ is the total particle number per volume of the slurry; $\mu_{3}$ is related to the total volume of crystals per volume of the slurry. Therefore, the volume-based mean crystal size $\left(L_{43}\right)$ and the coefficient of variation (CV) of the full CSD still can be captured by the MOM, as shown in (9) and (10).

$$
\begin{gathered}
L_{43}=\frac{\mu_{4}}{\mu_{3}} \\
C V=\sqrt{\frac{\mu_{5} \mu_{3}}{\mu_{4}^{2}}-1}
\end{gathered}
$$

Finally, after the optimization problem is solved based on the MOM model, the full CSD can be recovered by solving the full model of eqs.(1) and (2). In such a way, less-expensive function evaluations are possible for the optimization algorithms [12]. Care was taken to ensure that sufficient finite-volume grid points were selected [15] to obtain nearly identical results with MOM and FVM.

\section{OptimizATION OF MUlTi-SEGMENT MULTI-ADDition PLUG-FLOW CRYSTALLIZER}

Similar to cascaded multi-stage continuous MSMPR crystallizers [6], several segments of tubular crystallizer can also be joined together with temperature control applied to each segment, and additions of anti-solvent or fresh solution injected between any two consecutive segments, in order to efficiently regulate the solute concentration or solubility along the tube axial, as shown in Fig. 1. In the present study, each segment is regarded as a plug-flow crystallizer, which forms the multi-segment, multi-addition plug-flow crystallizer.

In terms of the simulation of the MSMA-PFC, the outlet slurry of one PFC segment is assumed to mix instantly with the added fresh solution or anti-solvent stream under an ideal mixing rule; hence the crystal number density $n$ and the solute concentration $C$ at the outlet should be diluted accordingly, and thus need to be updated and set as the boundary conditions for the next PFC segment, i.e., eqs.(4) and (5). For more information on this, readers are referred to [12].

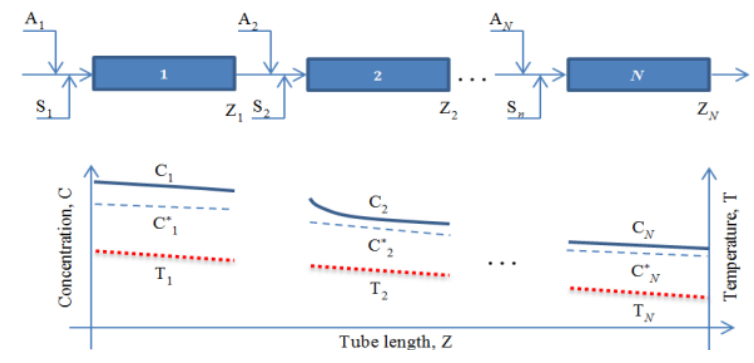

Figure 1. Schematic of a MSMA-PFC. $\left(\mathrm{A}_{i}\right.$ : anti-solvent addition for the $i$ th segment; $S_{i}$ : fresh solution addition; $Z_{i}$ : tube length; $C_{i}$ : solute concentration; $C^{*}$ : solute solubility; $T_{i}$ : temperature; $N$ : total number of segments.)
Previously, Alvarez et al. [1] reported a combination of four equal-length tubular units (length: $4 \times 0.6 \mathrm{~m}$; diameter: $1.27 \mathrm{~cm}$ ), with empirically designed distribution of anti-solvent among the four units, for example, $50 \%, 50 \%$, $0 \%, 0 \%$, and with a fixed amount of total anti-solvent addition. Ridder et al. [12] optimized the distribution of anti-solvent for a number of equally-spaced injection points, where up to 15 injection points were considered for a $50 \mathrm{~m}$ long tube, requiring a unit segment length of $50 / 15 \mathrm{~m}$. However, depending on the relative competitiveness of crystal growth rate and nucleation rate, varying ranges of supersaturation along the tubular crystallizer may be essential to achieve the best product attributes. Hence, by optimizing both the locations and distribution of a limited number of anti-solvent or fresh solution injections can lead to achieve better control of supersaturation with a smaller number of injection than using equal spatial distribution of the addition points.

To sum up accordingly, a practical multi-segment multi-addition tubular crystallizer should have the concept of modular design, which comprises standardized unit segments; for example, a module of $0.6 \mathrm{~m}$ long as in [1]. Then for a tubular crystallizer of $N$ segments, the locations and distribution of anti-solvent/fresh solution additions for a constant number of $m(m \leq N)$ injections points could be optimized. At the current stage, only the optimization of the anti-solvent addition is considered, as shown in eqs.(11)-(17) below; optimization of both anti-solvent and fresh solution additions would be straightforward and can be considered in future work.

$$
\begin{gathered}
\max _{\mathbf{U}, \mathbf{A}}(\mathbf{P})_{Z_{N}} \\
f(\mathbf{P})_{Z_{N}} \leq 0 \\
\mathbf{U}=\left[u_{2}, u_{3}, \ldots, u_{m}\right] \\
u_{i}=2, \ldots, N \\
u_{i}+1 \leq u_{i+1}, \quad i=2, \ldots, m-1 \\
\mathbf{A}=\left[x_{1}, x_{2}, \ldots, x_{m}\right] \\
\sum_{j=1}^{m} x_{j}=1, \quad j=1,2, \ldots, m
\end{gathered}
$$

where the product qualities $\mathbf{P}$ of the final segment at the exit position $Z_{N}$ are maximized, e.g., mean crystal size, product yield, etc.; linear and nonlinear constraints on product qualities $\mathbf{P}$ are summarized in (12), e.g., coefficient of variation; $\mathbf{U}$ is the integer vector for location indices, viz., $u_{i}$ means anti-solvent addition at the $u_{i}^{\text {th }}$ segment, since anti-solvent addition is always required for the first segment, $u_{1}=1$ and is not optimized; constraints (14) and (15) are to make sure that the indices are integers and in an ascending order; $\mathbf{A}$ is the vector of distribution fraction for each addition point under the assumption of a fixed amount of total anti-solvent addition, viz., $x_{j}$ is the distribution fraction for the jth segment.

Hence, under the proposed design and optimization framework, it is convenient to tailor a MSMA-PFC with a desired total length by assembling a number of unit segments 
[1] and also with an optimized anti-solvent distribution. Thus it is possible to make the MSMA-PFC flexible and efficient for various crystallization systems. Obviously, when a large number of injections are chosen, there would be only marginal benefits over the equally-spaced injections; hence, in practice $\mathbf{U}$ can be fixed in the optimization problem.

The above optimization problem of eqs.(11) to (17) is a mixed integer non-linear programming problem (MINLP) and can be solved by the genetic algorithm with an integer constraint "intcon" in MATLAB. The genetic algorithm is an adaptive heuristic search method based on the evolutionary ideas of natural selection and genetics and is capable of searching a large state-space or multi-modal state-space, offering significant benefits over more typical optimization techniques.

\section{RESULTS AND DISCUSSION}

\section{A. Tubular Crystallizer Design}

To demonstrate the proposed design and optimization framework for MSMA-PFC, a non-seeded anti-solvent crystallization system of paracetamol in acetone (solvent) and water (anti-solvent) mixture at a constant temperature of $16{ }^{\circ} \mathrm{C}$ is investigated in this study. The feeding fresh solution of paracetamol is first saturated at a water mass fraction of $60 \%$ and then injected into a $72 \mathrm{~m}$ long tube, which consists of 120 segments. Each modular segment is $0.6 \mathrm{~m}$ in length and $1.27 \mathrm{~cm}$ in diameter [1]. Addition of anti-solvent is also assumed to be possible only in the inlet of each modular segment. A total flow rate of $50 \mathrm{ml} \cdot \mathrm{min}^{-1}$ of fresh solution is fed to the first segment, and a total flow rate of $25 \mathrm{ml} \cdot \mathrm{min}^{-1}$ of water as anti-solvent is injected along the MSMA-PFC, the total residence time is approximated at about $120 \mathrm{~min}$, similar to the typical semi-batch crystallization process reported in [16], from which the solubility model and crystallization kinetics equations were adopted for case study purposes. It was observed that both crystal growth rate $G$ and nucleation rate $B$ are dependent on supersaturation and anti-solvent mass fraction; as previously mentioned the anti-solvent mass fraction has a complex effect on supersaturation, which make the control and optimization of product qualities very challenging. For example, see the anti-solvent addition flow rate profile by $\mathrm{C}$-control for semi-batch process in [16], where an exponential increase was required to maintain a constant trade-off of crystal growth and nucleation.

\section{B. Optimization of Anti-solvent addition}

Following the procedure proposed by Ridder et al., [12] a computationally efficient steady-state model of MSMA-PFC using MOM is employed first for the optimal design problem. The optimal results are then re-simulated by FVM to capture the full CSD development in the tubular crystallizer. To investigate the effect of the total number of injection points on the performance of the MSMA-PFC, anti-solvent addition is introduced through 1 to 6 injection points, with inlets at $m=1 \ldots 6$ segments are selected and injected with anti-solvent. In addition, three case studies are considered here for comparison purpose. Case 1, as a benchmark, considers equally-spaced injection points with equally distributed anti-solvent addition, as in [1]; Case 2 considers also the equally-spaced injection points, but with optimized distribution of anti-solvent addition, as in [12], where only vector $\mathbf{A}$ is optimized in the optimization problem of eq.(11) to achieve the largest volume-based mean crystal size $L_{43}$; while Case 3 optimizes both the location vector $\mathbf{U}$ and distribution vector $\mathbf{A}$ for the same objective function. In addition, for optimizations in Cases 2 and 3, constraints are imposed on the coefficient of variation $(\mathrm{CV} \leq 0.30)$ and the final paracetamol concentration $\left(C_{\mathrm{ZN}} \leq 0.806 \mathrm{~kg}\right.$ solute $/ \mathrm{kg}$ solvents), with an aim to maximize the mean crystal size $L_{43}$ without broadening the $\mathrm{CV}$ too much and with an acceptable yield.

The genetic algorithm for MINLP problem in MATLAB $2013 \mathrm{~b}$ was used for the optimization problems of Cases 2 and 3 . To cope with the stochastic nature of the genetic algorithm, population sizes of $30,60,100$ and 200 were implemented together with maximum generations of 150 for each optimization scenario of Cases 2 and 3; the best solution of each was then chosen as the final optimal result.

The final optimization results of the case studies are summarized in Table I. For example, in Case 2 of the MSMA-PFC with totally four injection points $(m=4)$, the location index vector $\mathbf{U}=\left[\begin{array}{llll}1 & 31 & 61 & 91\end{array}\right]$ means the third anti-solvent addition locates at the 61st segment, i.e. an axial position of $z=(61-1) \times 0.6 \mathrm{~m}=36.0 \mathrm{~m}$. The corresponding distribution vector $\mathbf{A}=[0.242,0.025,0.733,0.001]$ shows, for example, the amount of third anti-solvent addition is 0.733 $\times 25 \mathrm{ml} \mathrm{min}^{-1}=18.3 \mathrm{ml} \mathrm{min}^{-1}$. Comparisons of the three case studies for four injection points in MSMA-PFC are shown in Figs. 2 and 3 for concentration and the volume-based CSD profiles at specific axial positions, respectively.

Fig. 2 shows that all the three cases maintain a certain level of supersaturation, which reduces between additions; the first anti-solvent addition at the inlet of the crystallizer produces the first burst of nuclei which passes through the following PFC segments for further growth and finally contributes to the majority large particles at the outlet, as is demonstrated in Fig. 3. After the second addition, the equally-distributed addition in Case 1 creates a large supersaturation, which results in the second peak in the broadened CSD (see Fig. 3). Case 2 reduces the amount of anti-solvent addition at the same position compared to Case 1 at $z=18 \mathrm{~m}$; this extends the supersaturation level achieved in the previous segment, so that before the third addition (in which a large amount of anti-solvent is added to create high supersaturation) there are enough medium-size crystals to compete with the nucleation effect to consume the generated high supersaturation. In such a way, a significant increase of final mean crystal size $L_{43}$ from $369.7 \mu \mathrm{m}$ of Case 1 to 499.0 $\mu \mathrm{m}$ of Case 2 is observed in Table I, as well as a narrower CSD from Case 2. Interestingly, due to the limited residence time for crystal growth at the rear part of the crystallizer, there are only limited amounts of anti-solvent added from the last injection points close to the end of PFC, i.e., 91st, 97th, 101st in Case 2 (see Table I). Hence, when the total number of injection points is restricted, the optimal locations of the injection points are rather important.

Additional work by Ridder et al. [18] also demonstrated the effects of uncertainties in the accuracy of implementing the optimal antisolvent flow rates at each segment, which can have a significant effect on the CSD achieved in practice. 
TABLE I. SumMary OF THE THREE CASE STUdies

\begin{tabular}{clllccc}
\hline Case & $\mathbf{m}$ & \multicolumn{1}{c}{$\mathbf{U}$} & \multicolumn{1}{c}{$\mathbf{A}$} & $\boldsymbol{L}_{\mathbf{4 3}}(\boldsymbol{\mu m})$ & $\mathbf{C V}$ & $\boldsymbol{C}_{\mathbf{Z N}}(\mathbf{k g} / \mathbf{k g})$ \\
\hline & 1 & {$[1]$} & {$[1.000]$} & 278.5 & 0.182 & 0.0795 \\
& 2 & {$[161]$} & {$[0.500,0.500]$} & 273.3 & 0.160 & 0.0798 \\
1 & 3 & {$[14181]$} & {$[0.333,0.333,0.333]$} & 374.7 & 0.150 & 0.0804 \\
& 4 & {$[1316191]$} & {$[0.250,0.250,0.250,0.250]$} & 369.7 & 0.301 & 0.0806 \\
& 5 & {$[125497397]$} & {$[0.200,0.200,0.200,0.200,0.200]$} & 327.1 & 0.196 & 0.0808 \\
& 6 & {$[121416181101]$} & {$[0.166,0.166,0.166,0.166,0.166,0.166]$} & 361.5 & 0.188 & 0.0812 \\
& 1 & {$[1]$} & {$[1.000]$} & 278.5 & 0.182 & 0.0795 \\
& 2 & {$[161]$} & {$[0.245,0.755]$} & 494.9 & 0.251 & 0.0805 \\
2 & 3 & {$[14181]$} & {$[0.272,0.376,0.353]$} & 439.5 & 0.226 & 0.0806 \\
& 4 & {$[1316191]$} & {$[0.242,0.025,0.733,0.001]$} & 499.0 & 0.247 & 0.0805 \\
& 5 & {$[125497397]$} & {$[0.256,0.018,0.369,0.341,0.016]$} & 470.3 & 0.225 & 0.0806 \\
& 6 & {$[121416181101]$} & {$[0.233,0.035,0.079,0.546,0.106,0.001]$} & 483.5 & 0.229 & 0.0806 \\
& 1 & {$[1]$} & {$[1.000]$} & 278.5 & 0.182 & 0.0795 \\
& 2 & {$[1,62]$} & {$[0.246,0.754]$} & 497.2 & 0.248 & 0.0806 \\
& 3 & {$[14862]$} & {$[0.238,0.135,0.627]$} & 511.7 & 0.248 & 0.0806 \\
& 4 & {$[1465463]$} & {$[0.241,0.074,0.135,0.551]$} & 519.6 & 0.223 & 0.0806 \\
& 5 & {$[153616264]$} & {$[0.241,0.135,0.114,0.156,0.356]$} & 520.0 & 0.230 & 0.0806 \\
& 6 & {$[11950566367]$} & {$[0.237,0.003,0.071,0.111,0.516,0.063]$} & 521.1 & 0.238 & 0.0806 \\
\hline
\end{tabular}

In Case 3, both the locations and the distribution of the anti-solvent additions are optimized and as a result nearly constant supersaturation level is maintained up to the fourth addition at the $63^{\text {rd }}$ segment, after which large supersaturation level is also generated, analogous to that in Case 2. A further increase in the mean crystal size $L_{43}$, with a lower coefficient of variation is obtained for Case 3 compared to Case 2, as shown in Table I and Fig. 3. The latter indicates that Case 3 more successfully controls the nucleation effect throughout the whole tube.
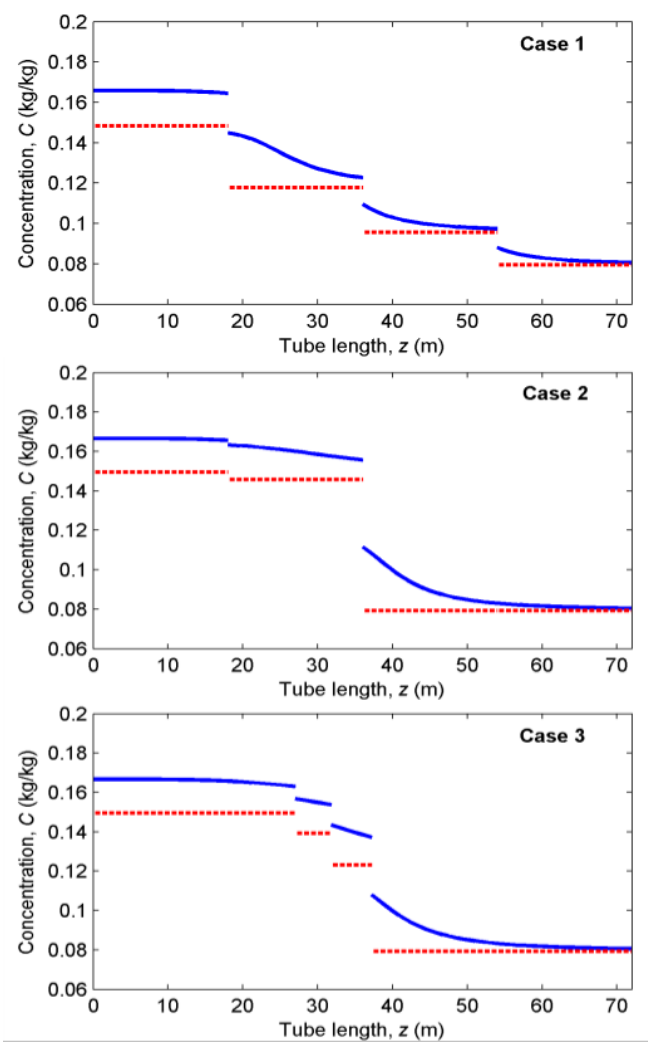

Figure 2. Optimization results of concentrations for four injection points in MSMA-PFC. (Solid line: solute concentration; dashed line: solute solubility.)
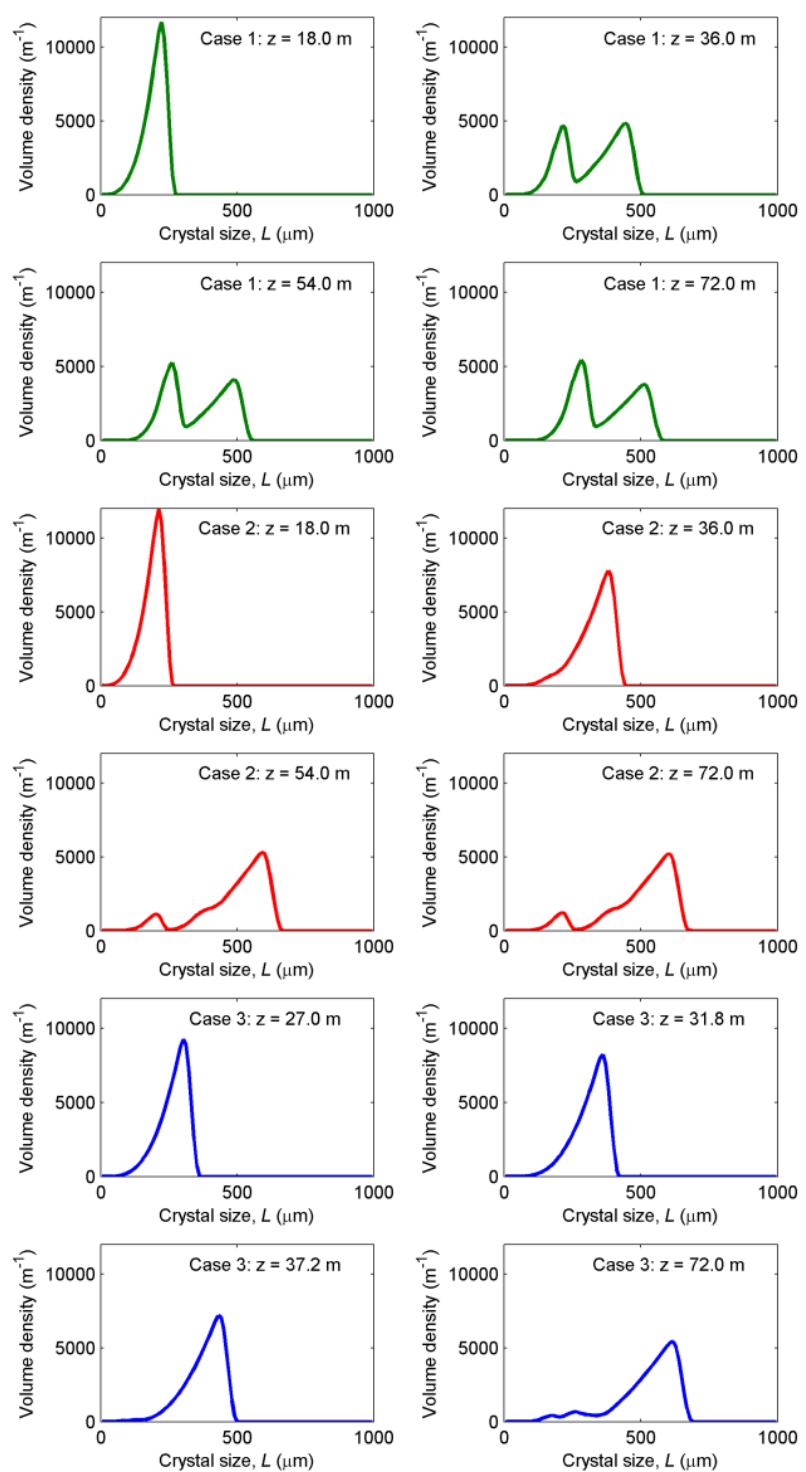

Figure 3. Optimization results of volume-based CSD for four injection points in MSMA-PFC (Upper four: Case 1; center four: Case 2; Lower four: Case 3). 


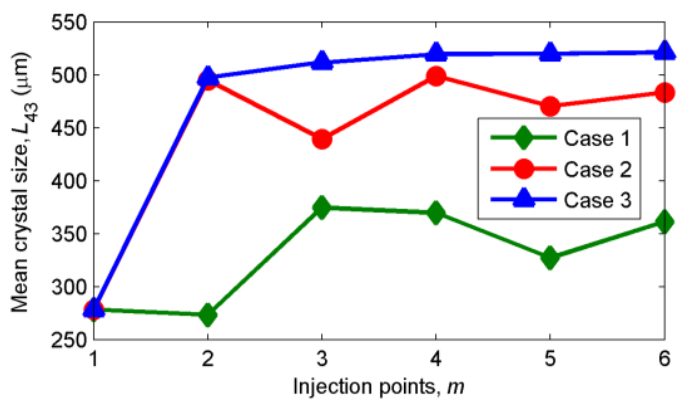

Figure 4. Effect of total number of injection points on optimization results of MSMA-PFC.

The effect of the total number of injection points is summarized in Table I and depicted in Fig. 4; Case 3 always produces the largest mean crystal size and increasing the number of injection points beyond four only contributes marginally to the performance of the MSMA-PFC. Importantly, consistent optimal locations for additions were found for Case 3, where nearly all of the anti-solvent is added in the first half of the crystallizer tube. However, variations in the optimization results were obtained for equally-spaced injection points of Cases 1 and 2, which were also reported in [12]. This is due to the fact that equally-spaced injection points add the anti-solvent more uniformly as the number of injection points increases; this strategy fails to provide enough anti-solvent addition in the first half of the tube, i.e. it does not inject the right amount of anti-solvent in the right place. As a result, optimization of both locations and amount of anti-solvent addition should be taken into account for MSMA-PFC to achieve an efficient and cost-effective design with desirable product attributes.

Incidentally, it is noted that the batch crystallization process with optimal C-control strategy and a seeded CSD (mean size $L_{43}=220 \mu \mathrm{m}$ ) achieved a final mean crystal size $L_{43}$ of $556 \mu \mathrm{m}$ under a batch time of $120 \mathrm{~min}$ in [16]. Therefore, the proposed design and optimization framework of MSMA-PFC shows the potential to implement an innovative unseeded continuous crystallizer design to retrofit an existing batch crystallization processes.

\section{CONCLUSION}

The MSMA-PFC has shown potential benefits for continuous crystallization processes in recent years. The current work has extended the previous work in mathematical modelling, design, and optimization of MSMA-PFC, proposing a conceptual design based on a number of standardized modular units and a corresponding optimization framework for optimizing locations and distributions of anti-solvent additions. Improvements to the previous optimization framework have been illustrated, as well the potential to replace existing batch crystallization processes. Future work would consider the effects of initial seeding at the inlet, additions of fresh solution along the tube, and a temperature profile for a combined cooling and anti-solvent crystallization process. Further extensions of the plug-flow crystalliser to a continuous oscillatory baffled crystallizer $(\mathrm{COBC})$ are also under investigation.

\section{REFERENCES}

[1] A. J. Alvarez and A. S. Myerson, "Continuous plug flow crystallization of pharmaceutical compounds," Cryst. Growth Des., vol. 10, no.5, pp.2219-2228, 2010

[2] A. Majumder and Z. K. Nagy, "Fines removal in a continuous plug flow crystalliser by optimal spatial temperature profiles with controlled dissolution," AIChE J., vol. 59, no. 12, pp.4582-4594, 2013.

[3] M. Kessel, "The problems with today's pharmaceutical business - an outsider's view," Nat. Biotechnol., vol. 29, no. 1, pp. 27-33, 2011

[4] S. Buchholz, "Future manufacturing approaches in the chemical and pharmaceutical industry," Chem. Eng. and Process., vol. 49, no. 10, pp. 993-995, 2010.

[5] B. Aksu, T. De Beer, S. Folestad, J. Ketolainen, H. Linden, J. A. Lopes, M. de Matas, W. Oostra, J. Rantanen and M. Weimer, "Strategic funding priorities in the pharmaceutical sciences allied to quality by design (QbD) and process analytical technology (PAT)," Eur. J. Pharm. Sci., vol. 47, no. 2, pp. 402-405, 2012.

[6] M. Sen, A. Rogers, R. Singh, A. Chaudhury, J. John, M. G. Ierapetritou and R. Ramachandran, "Flowsheet optimization of an integrated continuous purification-processing pharmaceutical manufacturing operation," Chem. Eng. Sci., vol. 102, pp. 56-66, 2013.

[7] S. Lawton, G. Steele, P. Shering, L. Zhao, I. Laird and X. W. Ni, "Continuous crystallization of pharmaceuticals using a continuous oscillatory baffled crystallizer," Org. Process Res. \& Dev., vol. 13, no. 6, pp. 1357-1363, 2009.

[8] R. J. P. Eder, S. Radl, E. Schmitt, S. Innerhofer, M. Maier, H. Gruber-Woelfler and J. G. Khinast, "Continuously seeded, continuously operated tubular crystallizer for the production of active pharmaceutical ingredients," Cryst. Growth \& Des., vol. 10, no. 5, pp. 2247-2257, 2010.

[9] R. J. P. Eder, E. K. Schmitt, J. Grill, S. Radl, H. Gruber-Woelfler and J. G. Khinast, "Seed loading effects on the mean crystal size of acetylsalicylic acid in a continuous-flow crystallization device," Cryst. Res. Technol., vol. 46, no. 3, pp. 227-237, 2011.

[10] R. Lakerveld, H. J. M. Kramer, A. I. Stankiewicz and J. Grievink, "Application of generic principles of process intensification to solution crystallization enabled by a task-based design approach," Chem. Eng. Process.: Process Intensification, vol. 49, no. 9, pp. 979-991, 2010.

[11] T. Vetter, C. L. Burcham and M. F. Doherty, "Regions of attainable particle sizes in continuous and batch crystallization processes," Chem. Eng. Sci., vol. 106, pp. 167-180, 2014.

[12] B. J. Ridder, A. Majumder and Z. K. Nagy, "Population balance model based multi-objective optimization of a multi-segment multi-addition (MSMA) continuous plug-flow antisolvent crystallizer," Ind. Eng. Chem. Res., vol. 53, no. 11, pp. 4387-4397, 2014.

[13] S. Byrn, M. Futran, H. Thomas, E. Jayjock, N. Maron, R. F. Meyer, A. S. Myerson, M. P. Thien and B. L. Trout, "Achieving continuous manufacturing for final dosage formation: challenges and how to meet them," in International Symposium on Continuous Manufacturing of Pharmaceuticals, Cambridge, May 20-21, 2014.

[14] H. Leuenberger, "New trends in the production of pharmaceutical granules: batch versus continuous processing," Eur. J. Pharm. Biopharm., vol. 52, no. 3, pp. 289-296, 2001.

[15] A. Mesbah, H. J. M. Kramer, A. E. M. Huesman and P. M. J. van de Hof, "A control oriented study on the numerical solution of the population balance equation for crystallization process," Chem. Eng. Sci., vol. 64, pp. 4262-4277, 2009.

[16] X. Y. Woo, Z. K. Nagy, R. B. H. Tan and R. D. Braatz, "Adaptive concentration control of cooling and antisolvent crystallization with laser backscattering measurement," Cryst. Growth Des., vol. 9, no. 1 , pp. 182-191, 2009.

[17] B. J. Ridder, A. Majumder and Z. K. Nagy, "Population balance model based multi-objective optimization of a multi-segment multi-addition (MSMA) continuous plug-flow antisolvent crystallizer," Ind. Eng. Chem. Res., vol. 53, no. 11, pp. 4387-4397, 2014.

[18] B.J. Ridder, A. Majumder, Z.K. Nagy, Uncertainty analysis and robust multi-objective optimization of a continuous plug flow crystallization system, in Proc. of the American Control Conference (ACC 2014), IEEE Press, Piscataway, NY, Seattle, USA, 2014. 